\title{
Expression of and correlational patterns among neuroinflammatory, neuropeptide, and neuroendocrine molecules from cerebrospinal fluid in cerebral palsy
}

\author{
Cory J. Goracke-Postle ${ }^{1}$, Chantel C. Burkitt ${ }^{2}$, Angela Panoskaltsis-Mortari ${ }^{3}$, Michael Ehrhardt ${ }^{3}$, George L. Wilcox ${ }^{4}$,
} Patrick Graupman ${ }^{2}$, Michael Partington ${ }^{5}$ and Frank J. Symons ${ }^{6^{*}}$

\begin{abstract}
Background: The underlying pathogenesis of cerebral palsy (CP) remains poorly understood. The possibility of an early inflammatory response after acute insult is of increasing interest. Patterns of inflammatory and related biomarkers are emerging as potential early diagnostic markers for understanding the etiologic diversity of CP. Their presence has been investigated in plasma and umbilical cord blood but not in cerebrospinal fluid (CSF).

Methods: A clinical CP sample was recruited using a single-time point cross-sectional design to collect CSF at pointof-care during a standard-of-care surgical procedure (intrathecal pump implant). Patient demographic and clinical characteristics were sourced from medical chart audit.

Results: Significant $(p \leq 0.001)$ associations were found among neuroinflammatory, neuroendocrine, and nociceptive analytes with association patterns varying by birth status (term, preterm, extremely preterm). When between birth-group correlations were compared directly, there was a significant difference between preterm and extremely preterm birth subgroups for the correlation between tumour necrosis factor alpha (TNFa) and substance P.

Conclusion: This investigation shows that CSF can be used to study proteins in CP patients. Differences in intercorrelational patterns among analytes varying by birth status underscores the importance of considering birth status in relation to possible mechanistic differences as indicated by biomarker signatures. Future work should be oriented toward prognostic and predictive validity to continue to parse the heterogeneity of CP's presentation, pathophysiology, and response to treatment.
\end{abstract}

Keywords: Cerebral palsy, Neuroinflammation, Neuroendocrine, Nociceptive, Biomarkers, CSF, Children

\section{Background}

Cerebral palsy (CP) is a neurodevelopmental disorder of movement, muscle tone or posture caused by an insult to the developing brain before birth, at birth, or before the

\footnotetext{
*Correspondence: symon007@umn.edu

${ }^{6}$ Department of Educational Psychology, College of Education

and Human Development, Minneapolis, MN 55455, USA

Full list of author information is available at the end of the article
}

age of two years. $\mathrm{CP}$ is the most common motor disability in childhood, affecting an estimated 500,000 American children and occurring in approximately 2 children per 1,000 live births. The pathogenesis of $\mathrm{CP}$ is not fully understood. CP risk factors predominantly involve perinatal factors such as anoxia and ischemia and prenatal factors such as young gestational age, intrauterine viral infections, and maternal thyroid abnormalities. Intrauterine infection and inflammation are of particular 
interest with both maternal response (chorioamnionitis) and fetal response (funicitis or elevated interleukin-6 in fetal plasma) being associated with white matter damage (WMD) and CP [1]. Injury and related inflammatory processes may persist for considerable periods of time (years) leading to hypotheses and emerging models of tertiary-like mechanism of damage including epigenetic regulation and inflammatory relevant changes [2].

Work investigating infection- and neuroinflammatoryrelated biomarkers related to WMD in neonates has generated evidence mostly supporting a pathway from intrauterine infection to placental inflammation then to systemic fetal circulation and the preterm newborn brain [3]. The specifics of the infection-inflammation-brain damage link have been described and documented and are an active area of investigation [4] particularly given cautions about what level of inference is or is not supported by high quality evidence [5]. What is less clear, however, is the robustness of the inference supporting an infection-inflammation-WMD link with $\mathrm{CP}$ as an inevitable outcome. There is some evidence for such a link [6], but it may be conditioned on whether the infant was born term or preterm. From a more general perspective, the emerging viewpoint on the role of neuro-inflammation as a pathophysiological contributor to $\mathrm{CP}$ has created the possibility of a new therapeutic window through which to view the condition $[7,8]$.

Specific findings from prior clinical work specifically investigating inflammatory mediators have demonstrated differential biomarker patterns in umbilical cord serum from infants stratified by preterm and $\mathrm{CP}$ status [1]. Of the potential inflammation markers that differed between cases and controls, the markers were lower (based on medians) in the preterm CP cases relative to controls but were higher, relative to controls, in the term CP cases. Sampling from a different compartment (plasma) and using a different approach, a study by Lin et al. (2010) comparing school-age children who were former preterm births reported higher cytokine responses (increased tumor necrosis factor-alpha $[$ TNF- $\alpha]$ plasma levels and greater mRNA levels of tolllike receptor four [TLR4]) among those children with $\mathrm{CP}$ who were preterm relative to controls (children born preterm with normal development) [9]. Another study of blood, using a serial approach (i.e., repeated measures) in over 900 preterm infants, documented elevated concentration values of myriad inflammatoryrelevant mediators which were associated with different risk profiles depending on the sampling day [10]. Using CSF from preterm infants with brain injury, DouglasEscobar and Weiss (2012) documented combinations of biomarker concentration values that could be used to inform clinical decision making [4]. Using blood from a sample of children with and without CP, Zareen (2020) [11] found significantly increased levels of erythropoietin at baseline in children with CP compared with children in the comparison group. In response to challenge (lipopolysaccharide), both groups had appropriate and comparable response profiles for interleukin-8, vascular endothelial growth factor (VEGF), TNF- $\alpha$, and granulocyte-macrophage colony-stimulating factor (GMCSF) levels. The children with CP showed a statistically significant lipopolysaccharide hypo-responsiveness profile for interleukin-1a, interleukin-1b, interleukin-2, and interleukin- 6 levels. Collectively, the work to date consistently shows immune and inflammatory differences in children with $\mathrm{CP}$.

There remain gaps in our knowledge about the specific linkages among various immune and related mechanisms driving hypothesized persistent inflammatory states in children with $\mathrm{CP}$, and the relation among the various biomarkers, risk factors, and specific outcomes. The overall generality of the findings to date for the CP population is limited by two kinds of problems, namely the relative difficulties in establishing valid preclinical models for this purpose [12] and the extreme paucity of clinically-relevant biomarker research within this high-need vulnerable patient group. For example, the relation among the CSF biomarkers investigated by Douglas-Escobar and Weiss (2012) and CP as an outcome was not clear [4]. More biomarker data of a comparable kind from the same and different compartments across the same and different age groups are needed. To the best of our knowledge, no comparable work has investigated inflammatory-relevant molecular biomarkers in CSF from children, adolescents, and young adults with $\mathrm{CP}$.

The purpose of this preliminary investigation was exploratory. The design was cross-sectional using a single time point for specimen collection from a clinical sample. There were two specific aims. The first aim was to document levels of inflammatory and related molecules in CSF in a sample primarily of school-age children with CP. To do so, for detectable analytes, participants were arrayed along each analyte's concentration gradient. The second aim was to examine clinically relevant grouping variables (e.g., CP severity, term/preterm birth) to identify any potentially relevant correlational patterns among the molecules. Our intent was to extend the work initiated by Kaukola and Lin (described above) using a clinical sample in which standard-of-care surgical interventions were leveraged to gain access to CSF for future hypothesis-generating research purposes. 


\section{Methods}

\section{Protocol approval}

This study was approved by the Institutional Review Board (IRB, \#0809M46301) of the University of Minnesota. Written informed consent was obtained from each participant or legal representative (i.e., parent/guardian).

\section{Participants}

This study utilized a single-time point cross-sectional design. Twenty-eight individuals $(82 \%$ male $)$ with $\mathrm{CP}$ participated (mean age $=9.74$ years, $\mathrm{SD}=4.36$; range $=4-23)$. Specific CP diagnoses included: quadriplegia $(n=17)$, diplegia $(n=5)$, and triplegia $(n=5)$. Participants were included in the study if they (a) had cerebral palsy, (b) were between 3-25 years of age, and (c) were scheduled for initial intrathecal baclofen (ITB) pump implant. Individuals were excluded if (a) they had an existing cerebral shunt; or (b) they had compounded dosing (i.e., opioid adjunctive to baclofen) through their pump. The participants were already characterized clinically using the Gross Motor Functional Classification System for Cerebral Palsy (GMFCS) to categorize gross motor function. The GMFCS is a 5-level classification system based on self-initiated movement with emphasis on truncal control and walking. The GMFCS is widely used clinically and in classifying individuals with CP for research studies [13].

For the subgroup comparisons, the breakdown of participant demographics was as follows: males $(n=23)$ and females $(n=5)$; non-quadriplegia $(n=11)$ and quadriplegia $(n=17)$; Caucasian $(n=26)$ and other $(n=2)$; spastic CP $(n=13)$ and mixed tone CP $(n=12)$; term birth, defined as 37 weeks or later $(n=6)$, preterm birth, defined as $28-37$ weeks $(n=12)$ and extremely preterm birth, defined as less than 28 weeks $(n=9)$; seizure $(n=6)$ and no seizure $(n=16)$ (Table 1$)$.

\section{CSF collection}

Patients were consented in accordance with an approved IRB protocol. If consent was given, CSF was collected during a standard-of-care surgical procedure (ITB pump implant). In all cases, the surgery proceeded as usual until the spinal catheter had been placed. Then, the neurosurgeon collected 10-20 $\mathrm{ml}$ of CSF from the spinal catheter placed well above the spinal puncture site. This method avoided contaminating the collected CSF with blood.

Immediately following collection, the CSF was placed on wet ice $\left(+4{ }^{\circ} \mathrm{C}\right)$ and transported to a cold room for processing, centrifuged at $3000 \mathrm{rpm} \times 5 \mathrm{~min}$, pipetted into 100 and $250 \mu \mathrm{L}$ aliquots, flash frozen in liquid nitrogen and archived at $-80{ }^{\circ} \mathrm{C}$. After specimen collection, the patient was monitored closely following routine operative and post-operative procedures. There were no adverse events.

\section{CSF analyte analysis}

CSF was analyzed using conventional biochemical methods based on commercially available enzyme-linked immunosorbent assay (ELISA) kits and expression levels of each marker were quantified. Specifically, samples were tested by the Cytokine Reference Laboratory (CRL, University of Minnesota). This is a CLIA'88-licensed facility (license \#24D0931212). Samples were analyzed for adrenocorticotropic hormone (ACTH), agoutirelated peptide (AgRP), brain-derived neurotrophic factor (BDNF), ciliary neurotrophic factor (CNTF), follicle-stimulating hormone (FSH), growth hormone $(\mathrm{GH})$, luteinizing hormone $(\mathrm{LH})$, prolactin (PRL), and thyroid stimulating hormone (TSH) using the "Human Brain-Derived Protein Panel" on the Luminex platform and done as a multi-plex (Luminex instrument-Bioplex 100 [Bio-Rad, 1000 Alfred Nobel Drive, Hercules, CA, 94547], Software: bio-plex Manager 4.0). The polystyrene bead set (cat. \# HPT-66 K-09) with kit lot number 1757143 was used. Kits/reagents were purchased from EMD Millipore Corporation, Billerica, MA. Interferon $\alpha 2$ (IFN $\alpha 2)$, interleukin-1 $\alpha$ (IL-1 $\alpha)$, interleukin-1ra (IL-1ra), interleukin-6 (IL-6), interleukin-8 (IL-8), interleukin-10 (IL-10), interleukin-12p40 (IL-12p40), interleukin-12p70 (IL-12p70), interferon gamma-induced protein 10 (IP$10)$, monocyte chemotactic protein (MCP-1), macrophage inflammatory protein $1 \beta$ (MIP1 $\beta)$, regulated on activation normal $\mathrm{T}$ expressed and secreted (RANTES), and tumor necrosis factor $\alpha(\mathrm{TNF} \alpha)$ were analyzed using the "Cytokine/Chemokine Panel 1" on the Luminex platform and done as a multi-plex (Luminex instrumentBioplex 100 [Bio-Rad, 1000 Alfred Nobel Drive, Hercules, CA, 94547], Software: bio-plex Manager 4.0). The polystyrene bead set (cat. \# MPXHCYTO-60 K-14) with kit lot number 1757142 was used. Kits/reagents were purchased from EMD Millipore Corporation, Billerica, MA. Dynorphin A, neuropeptide $Y$, somatostatin, $\beta$ endorphin, cortisol, neurotensin, orexin A, substance P, melatonin, oxytocin, and melanocyte-stimulating hormone $(\alpha-\mathrm{MSH})$ were analyzed using the "Human Neuropeptide Panel" on the Luminex platform and done as a multi-plex (Luminex instrument-Bioplex 100 Bio-Rad, 1000 Alfred Nobel Drive, Hercules, CA, 94547], Software: bio-plex Manager 4.0). The polystyrene bead set (cat. \# HNP35 K-08) with kit lot number 1823005 was used. Kits/reagents were purchased from EMD Millipore Corporation, Billerica, MA.

Samples were assayed according to manufacturer's instructions. ELISA employ the quantitative sandwich 
Table 1 Participant health information; $M \pm S D$ or $n(\%)$

\begin{tabular}{|c|c|c|c|c|}
\hline & $\begin{array}{l}\text { Complete sample } \\
(n=28)\end{array}$ & $\begin{array}{l}\text { Term birth } \\
(n=6)\end{array}$ & $\begin{array}{l}\text { Preterm birth } \\
(n=12)\end{array}$ & $\begin{array}{l}\text { Extremely preterm birth } \\
(n=9)\end{array}$ \\
\hline Male & $23(82.1)$ & $3(50.0)$ & $11(91.7)$ & $9(100)$ \\
\hline \multicolumn{5}{|l|}{ Ethnicity } \\
\hline Caucasian & $26(92.9)$ & $6(100.0)$ & $12(100.0)$ & $8(88.9)$ \\
\hline African American & $1(3.6)$ & $0(0)$ & $0(0)$ & $1(11.1)$ \\
\hline Other (not specified) & $1(3.6)$ & $0(0)$ & $0(0)$ & $0(0)$ \\
\hline \multicolumn{5}{|l|}{ Epilepsy } \\
\hline None & $16(57.1)$ & $3(50.0)$ & $7(58.3)$ & $6(66.7)$ \\
\hline History of seizure/diagnosis of epilepsy & $6(21.4)$ & $1(16.7)$ & $4(33.3)$ & $1(11.1)$ \\
\hline Questionable seizure activity & $3(10.7)$ & $1(16.7)$ & $0(0)$ & $1(11.1)$ \\
\hline Missing & $3(10.7)$ & $1(16.7)$ & $1(8.3)$ & $1(11.1)$ \\
\hline \multicolumn{5}{|l|}{ CP topography } \\
\hline Hemiplegia & $1(3.6)$ & $0(0)$ & $1(8.3)$ & $0(0)$ \\
\hline Diplegia & $5(17.9)$ & $2(33.3)$ & $3(25.0)$ & $0(0)$ \\
\hline Triplegia & $5(17.9)$ & $1(16.7)$ & $0(0)$ & $4(44.4)$ \\
\hline Quadriplegia & $17(60.7)$ & $3(50.0)$ & $8(66.7)$ & $5(55.6)$ \\
\hline \multicolumn{5}{|l|}{ GMFCS } \\
\hline Level I & $3(10.7)$ & $0(0)$ & $3(25.0)$ & $0(0)$ \\
\hline Level II & $3(10.7)$ & $1(16.7)$ & $0(0)$ & $2(22.2)$ \\
\hline Level III & $7(25.0)$ & $3(50.0)$ & $2(16.7)$ & $2(22.2)$ \\
\hline Level IV & $8(28.6)$ & $1(16.7)$ & $4(33.3)$ & $3(33.3)$ \\
\hline Level V & $6(21.4)$ & $1(16.7)$ & $2(16.7)$ & $2(22.2)$ \\
\hline Missing & $1(3.6)$ & $0(0)$ & $1(8.3)$ & \\
\hline \multicolumn{5}{|l|}{ Tone } \\
\hline Spastic & $13(46.4)$ & $4(66.7)$ & $5(41.7)$ & $4(44.4)$ \\
\hline Mixed tone & $12(42.9)$ & $2(33.3)$ & $5(41.7)$ & $4(44.4)$ \\
\hline Missing & $3(10.7)$ & $0(0)$ & $2(16.7)$ & $1(11.1)$ \\
\hline \multicolumn{5}{|l|}{ Current feeding tube } \\
\hline Yes & $8(28.6)$ & $2(33.3)$ & $2(16.7)$ & $4(44.4)$ \\
\hline No & $19(67.9)$ & $4(66.7)$ & $10(83.3)$ & $5(55.5)$ \\
\hline Missing & $1(3.6)$ & $0(0)$ & $0(0)$ & $0(0)$ \\
\hline $\begin{array}{l}\text { Days in NICU at birth } \\
\text { Range of NICU days }\end{array}$ & $\begin{array}{l}76.25 \pm 67.04 \\
(0-300)\end{array}$ & $\begin{array}{l}3.50 \pm 7.00 \\
(0-14)\end{array}$ & $\begin{array}{l}52.73 \pm 29.72 \\
(10-120)\end{array}$ & $\begin{array}{l}137.33 \pm 64.76 \\
(90-300)\end{array}$ \\
\hline
\end{tabular}

Note: gestational age was not available for one participant; Term birth = born 37 weeks gestation or later; preterm birth = born at 28-37 weeks gestation; extremely preterm birth = born at less than 28 weeks gestation; CPCerebral Palsy, GMFCS Gross Motor Function Classification System, level / ambulant without assistance, level II ambulant without assistive devices, limitations outside the home, level III ambulant with assistive devices, wheelchair required outside the home, level IVnonambulatory, self-mobile in wheelchair with limitations, level $V$ non-ambulatory, self-mobility very limited

enzyme immunoassay technique. The absorbance is measured on the microtiter plate reader (Bio-Rad model 550). The intensity of the color formed is proportional to the concentration of the sample. Fluorescent colorcoded beads coated with a specific capture antibody were added to each sample. After incubation, and washing, biotinylated detection antibody was added, followed by phycoerythrin-conjugated streptavidin. The beads were read on a Luminex instrument (Bioplex 100) which is a dual-laser fluidics-based instrument. One laser determines the analyte being detected via the color coding; the other measures the magnitude of the PE signal from the detection antibody which is proportional to the amount of analyte bound to the bead. Samples were tested in duplicate and values were interpolated from 5 parameterfitted (5PL) standard curves.

\section{Statistical analyses}

Data analysis was exploratory and relied on visual analysis, descriptive statistics, and correlational analyses. First, visual analysis of each analyte was conducted to understand its distributional form and to identify potential outliers. Further, measures of central tendency (means, 
medians) and variation (standard deviations, coefficients of variation) were calculated for each analyte.

Second, to understand the associations between analytes a series of pairwise scatterplots and Pearson product-moment correlations were computed between each possible pair of analytes for the entire sample. The correlations were tested for statistical significance against the null hypothesis of $r=0$. Parallel analyses and plots were generated for the data set with missing data imputed with the lower limit / sensitivity number. Given the large number of correlations tested (528), Type 1 errors were controlled for by using the false discovery rate correction discussed by Benjamini \& Hochberg (1995). Correlations were considered against an alpha $=0.05$, after the false discovery rate correction was applied.

Third, to understand how the associations between analytes varied by subgroups, the correlational analyses described above were repeated for each of the gestational term subgroups. Given the large number of comparisons involved in these analyses, an alpha $=0.001$ was set for each test after the false discovery correction rate was applied.

Finally, differences in correlations between subgroups were also tested. To be included in between-group analysis, each correlation first had to be statistically significant within the subgroups. Thirty-two pairs of correlations were statistically significant across all subgroups. Then the identified correlations were tested against one another to check whether they were significantly different by birth status. To do this, Fisher's $r$ to $\mathrm{z}$ transformation was used to calculate the difference in correlations that met the criteria for inclusion, and tested for statistical significance of that difference; $\mathrm{p} \leq 0.05$.

\section{Results}

CSF analyte expression in CP subjects

Detectable CSF analytes were broken down, broadly, by the following categories: hormonal/endocrine brain-derived peptides or proteins: ACTH, AgRP,

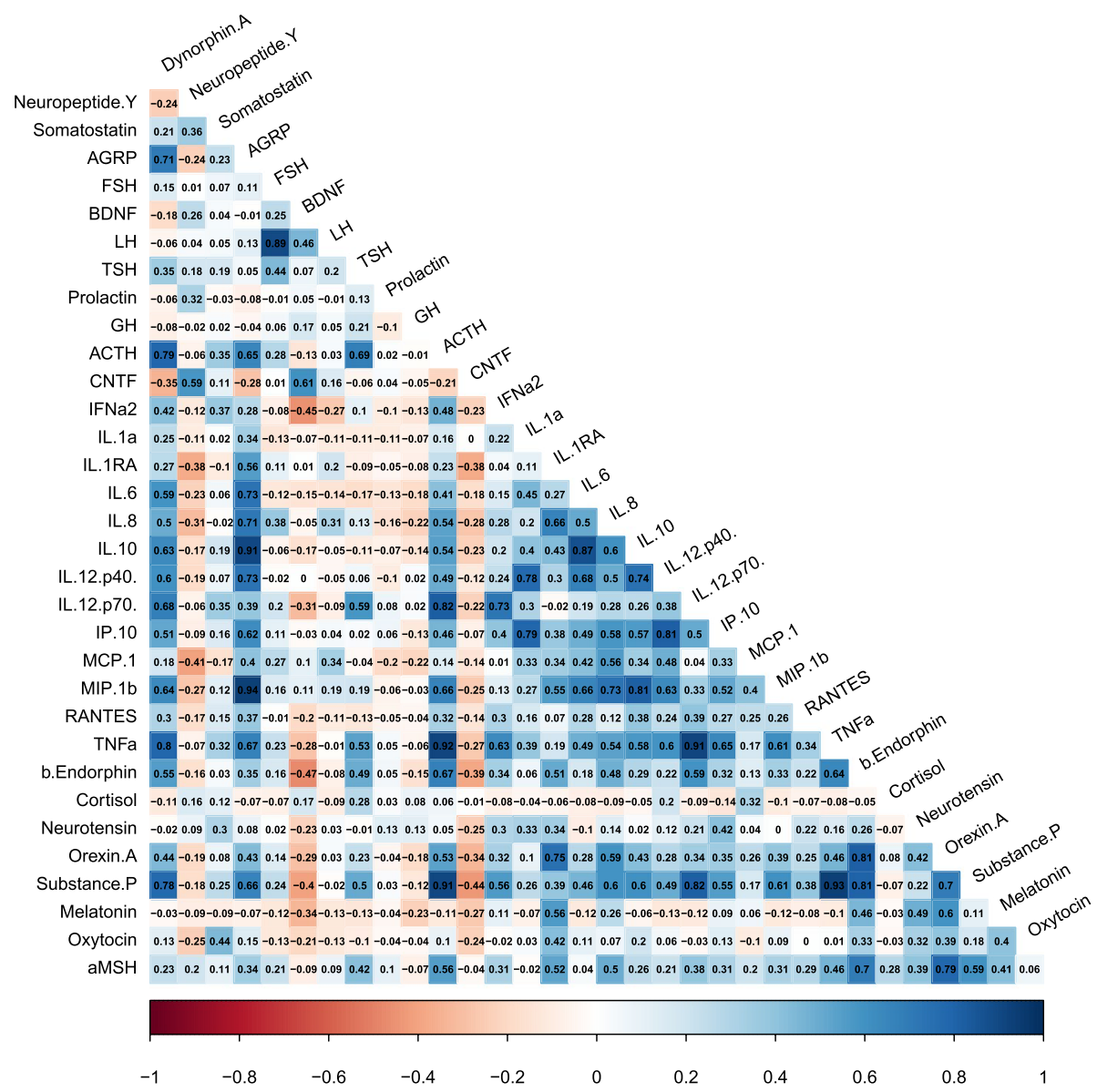

Fig. 1 Visual representation of the direction and strength of the Pearson's correlation coefficients between all 33 analytes assayed. Positive (blue), negative (red), strong (dark shading), and weak (light shading) correlations are depicted 
BDNF, CNTF, FSH, GH, LH, PRL, and TSH; inflammatory cytokines/chemokines: IL-1 $\alpha$, IL-1ra, IL-6, IL-8, IL-10, IL-12p40, IL-12p70, TNF $\alpha$, IFN- $\alpha 2$, IP-10, MCP-1, MIP1 $\beta$, and RANTES; and neurotransmitters/ neuropeptides: Dynorphin A, neuropeptide Y, somatostatin, $\beta$ endorphin, cortisol, neurotensin, orexin $A$, substance $\mathrm{P}$, melatonin, oxytocin, melanocyte-stimulating hormone $(\alpha-\mathrm{MSH})$. Figure 1 illustrates the correlations among analytes (at $p \leq 0.001$ ); a full list of analytes that demonstrated correlations $(p \leq 0.05)$ among the participants with CP is included in the Supplemental Information (Supplemental Table 1).

Considering this initial complete cohort, there were 35 analyte pairings with positive correlations as per our criteria from 21 distinct analytes (all $p \leq 0.001$; Table 2). These 35 correlations represent combinations of endocrine, inflammatory, and excitatory neuropeptides. The specific correlation pattern represents a novel approach to considering analytes that may shed light on the mechanistic underpinnings of secondary processes that may be ongoing in $\mathrm{CP}$ and result in clinical signs and their manifestation.

To assess the potential of such protein signatures in CSF to distinguish differences relating to biological variables underlying various subpopulations of CP patients, we assessed analyte correlations between various subgroups. This analysis provided novel information, with analyte signature correlations becoming apparent for specific subsets of participant groups. This report focuses on birth term as a defining characteristic; however, the Supplemental Information provides full analyses of various subgroups based on additional clinical characteristics.

\section{Gestational age subgroup analyses Term birth}

No significant correlations between analytes were detected at the pre-determined significance level $(p \leq 0.001)$ used to report the rest of the subgroup findings. There were, however, two correlations at $p \leq 0.05$ specific to Term Birth participants (correlations which were not present for Preterm Birth or Extremely Preterm Birth participants) specifically between IL-1ra and orexin A and between orexin A and substance P (identical data for both correlations: Correlation (Corr) $=0.99$; $95 \%$ Confidence Interval $(\mathrm{CI})=[0.89,1]$; Adjusted P (Adj P) $=0.041)$ (Fig. 2).

\section{Preterm birth}

Preterm Birth status resulted in clusters of analytes that were highly correlated to one another (Fig. 3). There were 14 discrete correlations not found in the other gestational subgroup (bolded in Table 3); these correlations
Table 2 Significant Pearson's correlations $(p \leq 0.001)$ between analyte pairs, representing 21 distinct analyte correlations (bold font)

\begin{tabular}{|c|c|c|c|c|}
\hline Analyte 1 & Analyte 2 & Correlation & $95 \% \mathrm{Cl}$ & Adj. $p$ \\
\hline Dynorphin A & AGRP & 0.71 & {$[0.34,0.85]$} & $<.001$ \\
\hline Dynorphin A & АCTH & 0.79 & {$[0.60,0.90]$} & $<.001$ \\
\hline Dynorphin A & IL-12p70 & 0.68 & {$[0.41,0.84]$} & 0.001 \\
\hline Dynorphin A & TNFa & 0.80 & {$[0.60,0.90]$} & $<.001$ \\
\hline Dynorphin A & substance $P$ & 0.78 & {$[0.57,0.89]$} & $<.001$ \\
\hline AGRP & IL-6 & 0.73 & {$[0.49,0.87]$} & $<.001$ \\
\hline AGRP & IL-8 & 0.71 & {$[0.46,0.86]$} & $<.001$ \\
\hline AGRP & IL-10 & 0.91 & {$[0.81,0.96]$} & $<.001$ \\
\hline AGRP & IL-12p40 & 0.73 & {$[0.50,0.87]$} & $<.001$ \\
\hline AGRP & MIP-1 $\beta$ & 0.94 & {$[0.87,0.97]$} & $<.001$ \\
\hline AGRP & TNFa & 0.67 & {$[0.40,0.84]$} & 0.001 \\
\hline FSH & LH & 0.89 & {$[0.78,0.95]$} & $<.001$ \\
\hline TSH & ACTH & 0.69 & {$[0.42,0.84]$} & 0.001 \\
\hline ACTH & IL-12p70 & 0.82 & {$[0.65,0.92]$} & $<.001$ \\
\hline ACTH & TNFa & 0.92 & {$[0.83,0.96]$} & $<.001$ \\
\hline ACTH & $\beta$ endorphin & 0.67 & {$[0.40,0.83]$} & 0.001 \\
\hline ACTH & substance P & 0.91 & {$[0.81,0.96]$} & $<.001$ \\
\hline IFNa2 & IL-12p70 & 0.73 & {$[0.49,0.87]$} & $<.001$ \\
\hline IL-1a & IL-12p40 & 0.78 & {$[0.57,0.89]$} & $<.001$ \\
\hline IL-1a & IP-10 & 0.79 & {$[0.60,0.90]$} & $<.001$ \\
\hline IL-1RA & orexin A & 0.75 & {$[0.52,0.88]$} & $<.001$ \\
\hline IL-6 & $\mathrm{IL}-10$ & 0.87 & {$[0.74,0.94]$} & $<.001$ \\
\hline IL-6 & IL-12p40 & 0.68 & {$[0.41,0.84]$} & 0.001 \\
\hline |L-8 & MIP-1 $\beta$ & 0.73 & {$[0.49,0.87]$} & $<.001$ \\
\hline IL-10 & IL-12p40 & 0.74 & {$[0.51,0.87]$} & $<.001$ \\
\hline IL-10 & MIP-1 $\beta$ & 0.81 & {$[0.63,0.91]$} & $<.001$ \\
\hline IL-12p40 & IP-10 & 0.81 & {$[0.63,0.91]$} & $<.001$ \\
\hline IL-12p70 & TNFa & 0.91 & {$[0.81,0.96]$} & $<.001$ \\
\hline IL-12p70 & substance $P$ & 0.82 & {$[0.64,0.91]$} & $<.001$ \\
\hline TNFa & substance $P$ & 0.93 & {$[0.86,0.97]$} & $<.001$ \\
\hline$\beta$ endorphin & orexin $A$ & 0.81 & {$[0.63,0.91]$} & $<.001$ \\
\hline$\beta$ endorphin & substance P & 0.81 & {$[0.62,0.91]$} & $<.001$ \\
\hline$\beta$ endorphin & aMSH & 0.70 & {$[0.44,0.85]$} & 0.001 \\
\hline orexin A & substance $P$ & 0.70 & {$[0.44,0.85]$} & 0.001 \\
\hline orexin A & aMSH & 0.79 & {$[0.58,0.90]$} & $<.001$ \\
\hline
\end{tabular}

represent unique correlations in this sample specific to individuals born preterm.

\section{Extremely preterm birth}

Extremely Preterm Birth status also resulted in distinct analyte correlations different from both Term Birth and Preterm Birth participants (Fig. 4). There were 24 discrete analyte correlations unique in this sample of participants in the extremely preterm birth status (bold in Table 4). 


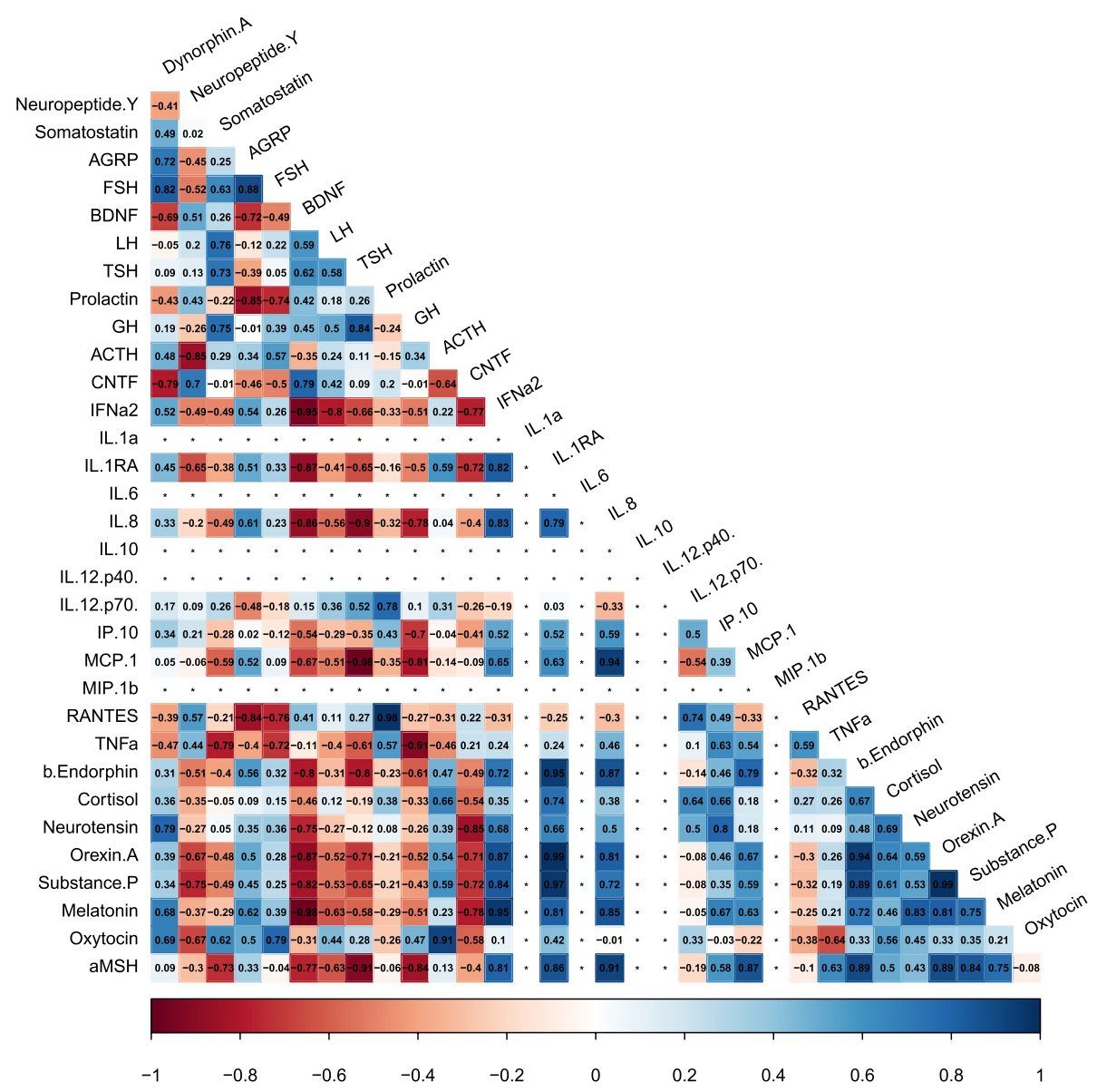

Fig. 2 Visual representation of the direction and strength of the Pearson's correlation coefficients between all 33 analytes assayed within the Term Birth subgroup. Positive (blue), negative (red), strong (dark shading), and weak (light shading) correlations are depicted. *In instances where the same value was reported for each variable, no correlation was calculated

\section{Between subgroup analyses}

Differences in correlations between subgroups were also directly tested. As noted above, to be included in between-group analysis, each correlation first had to be statistically significant within the subgroups. Thirtytwo pairs of correlations were statistically significant across all subgroups. Then the identified correlations were tested against one another to check whether they were significantly different by birth status. Based on this approach, there was a significant difference between the Preterm and Extremely Preterm Birth subgroups for the correlation between TNF $\alpha$ and substance P (Extremely Preterm $\mathrm{r}$ to $\mathrm{z}=0.99$, Preterm $\mathrm{r}$ to $\mathrm{z}=0.82$, $\mathrm{Z}$ Difference $=1.49, p<0.05$ ).

\section{Discussion}

There are many different ways that white matter and upper motor neurons can be damaged. It is generally agreed that enough damage will interfere with and ultimately impair motor control and increase risk for the clinical condition of CP (importantly there are, of course, other associated impairments including cognitive and sensory function). Of the many putative causal agents and pathways to $\mathrm{CP}$, the role of neuroinflammation in perinatal brain damage has received considerable empirical attention. There has also been considerable conceptual emphasis challenging conventional wisdom about the nature of the threat in relation to inflammation and the developing brain with a distinct shift to a perspective that a core underlying feature may be less about static events (i.e., a one-time insult) and more about sustained states (i.e., an ongoing process). The significance of this shift is in widening the focus of inquiry to include tertiary mechanisms of brain damage, which, in turn, could shed new light on a very old problem - namely treating $\mathrm{CP}$ based on its underlying pathophysiology.

There is a working hypothesis that suggests that the relationship among key neuroendocrine hormones, 


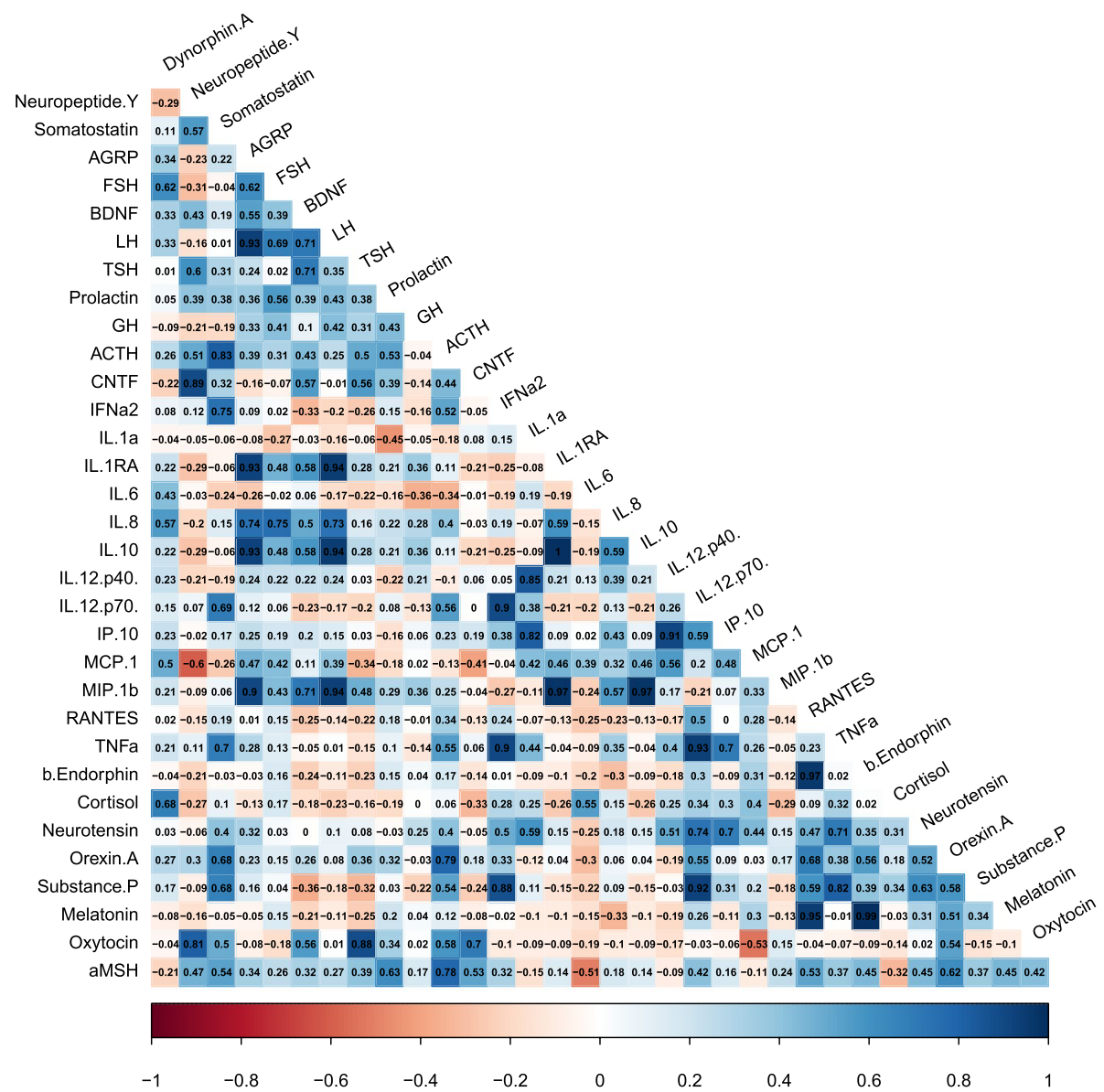

Fig. 3 Visual representation of the direction and strength of the Pearson's correlation coefficients between all 33 analytes assayed within the Preterm Birth subgroup. Positive (blue), negative (red), strong (dark shading), and weak (light shading) correlations are depicted

excitatory neuropeptides, and neuroinflammatory cytokines as markers may be a critical variable predicting outcome. The conceptual basis for this is the past two decades of work supporting the cross-talk among the endocrine, nociceptive, and immune systems. In healthy states there is an optimal balance between stress hormones and proinflammatory cytokines. In children with $\mathrm{CP}$, the white matter damage may be 'driving' an immune-mediated inflammatory cascade. There is evidence supporting this possibility from three related studies with CP patients, one using umbilical cord serum [1], one using plasma [9], and one using blood [11].

The current analysis expands the previous work and provides further description of the molecular milieu present in the CNS of CP patients. Such documentation provides a unique opportunity to consider how differences in CNS concentrations of various inflammatory-relevant analytes between differing presentations of $\mathrm{CP}$ may be relevant for hypotheses about mechanisms underlying the differential outcomes in CP. Specifically, in this sample, it was observed that Term Birth, Preterm Birth, and Extremely Preterm Birth status was associated with distinct patterns of analyte inter-correlations. If reproducibility of these findings could be established, there is an opportunity to better understand the mechanisms underlying various and varying outcomes specific to birth term in CP.

The correlations present in the participants suggest relationships among systems subserving arousal (orexin A), inflammation (TNF $\alpha$ ), anti-inflammation (IL-1ra), and neuronal excitation (substance $\mathrm{P}$ ), with distinctions depending on birth term. Orexin A (hypocretin 1) is an excitatory, hypothalamic neuropeptide that binds to both the orexin 1 and 2 receptors (OX1R and OX2R); the former is thought to act largely through the excitatory $\mathrm{Gq}$ protein and both are implicated in wakefulness and sleep cycle stability [14]. Narcoleptic symptoms in dogs and mice are associated with loss-of-function mutations in the gene encoding OX2R, although immunosuppression can delay symptom onset, and deletion of the gene 
Table 3 Significant Pearson's correlations $(p \leq 0.001)$ between analyte pairs in the Preterm Birth subgroup are displayed. There were 14 unique positive analyte correlations (bold font) specific to the Preterm Birth subgroup that were not significant within the other gestational age subgroups

\begin{tabular}{lllll}
\hline Analyte 1 & Analyte 2 & Correlation & $95 \% \mathrm{Cl}$ & Adj. $\mathrm{p}$ \\
\hline AGRP & IL-1 ra & 0.93 & {$[0.75,0.98]$} & 0.001 \\
AGRP & IL-10 & 0.93 & {$[0.75,0.98]$} & 0.001 \\
IL-12.p40 & IP-10 & 0.91 & {$[0.70,0.97]$} & 0.001 \\
IL-12.p70 & substance P & 0.92 & {$[0.73,0.98]$} & 0.001 \\
AGRP & LH & 0.93 & {$[0.77,0.98]$} & $<.001$ \\
LH & IL-1 ra & 0.94 & {$[0.80,0.98]$} & $<.001$ \\
LH & IL-10 & 0.94 & {$[0.80,0.98]$} & $<.001$ \\
LH & MIP-1 $\beta$ & 0.94 & {$[0.79,0.98]$} & $<.001$ \\
IL-1ra & IL-10 & 1.00 & {$[1.00,1.00]$} & $<.001$ \\
IL-1ra & MIP-1 $\beta$ & 0.97 & {$[0.90,0.99]$} & $<.001$ \\
IL-10 & MIP-1 $\beta$ & 0.97 & {$[0.90,0.99]$} & $<.001$ \\
IL-12.p70 & TNFa & 0.93 & {$[0.78,0.98]$} & $<.001$ \\
RANTES & $\beta$ endorphin & 0.97 & {$[0.89,0.99]$} & $<.001$ \\
RANTES & Melatonin & 0.95 & {$[0.81,0.99]$} & $<.001$ \\
$\beta$ endorphin & Melatonin & 0.99 & {$[0.97,1.00]$} & $<.001$ \\
\hline
\end{tabular}

encoding both orexins in mice results in a full narcolepsy phenotype [14]. Substance P is another excitatory neuropeptide that binds to neurokinin 1 receptors (NK1R), which couple through the excitatory Gq protein. Substance $\mathrm{P}$ is contained in and released by some $\mathrm{C}$-fiber nociceptors and causes activation and internalization of NK1Rs on nociceptive spinal cord neurons, exciting them [15]. These neurons project to the thalamus carrying information on the sensory-discriminative aspects of pain ultimately to the somatosensory cortex [16]. Thus, Substance $\mathrm{P}$ is thought to be one of the important drivers of pain transmission in spinal cord and trigeminal nucleus. IL-1ra is an endogenous anti-inflammatory cytokine that binds unproductively to the IL-1 receptor 1 (IL1R1), thereby blocking signaling by two proinflammatory cytokines IL- $1 \alpha$ and IL- $1 \beta$. The FDA has approved an altered form of human IL-1ra in the form of anakinra for use in peripheral inflammatory disorders like rheumatoid arthritis [17]. In the CNS, both of these cytokines, acting through IL1R1s on astrocytes, endothelial cells, and neurons, initiate transcription of multiple pro-inflammatory cytokines, including TNF $\alpha$, leading to reactive gliosis and enhanced neuronal excitability following such insults as traumatic brain injury.

In clinical investigations, evidence suggests an autoimmune destruction of orexin neurons [14], and orexin A and IL-1ra in CSF have been associated with fatigue in Sjögren's syndrome [18]. At least one study found decreased levels of substance $\mathrm{P}$ in the CSF of patients with narcolepsy [19], a disorder for which orexin A deficiency is a well-known biomarker [20]. Sleep dysfunction is well-established in CP; our observations suggest that specific inflammatory and nociceptive mediators may be implicated and future work should be designed to investigate in more detail the relation between sleep, inflammation, and neuronal excitation in $\mathrm{CP}$ based on biomarker-informed molecular signatures that may be specific to birth status.

Further, the relevance of the difference between the Preterm and Extremely Preterm subgroups appears particularly striking and significant given the literature documenting the clinical differences in phenotypic presentation between these two groups. Understanding the relationship between TNF $\alpha$ and substance P expression and regulation may have implications related to the underlying pathophysiology with possible prognostic and treatment relevance. One study of cultured human astrocytes demonstrated a functional interaction between these two analytes: substance $\mathrm{P}$ enhanced the stimulatory effect of TNF $\alpha$ on production of two inflammatory mediators: IL-6 and PGE ${ }_{2}$ [21]. This birth term-associated analyte difference may underscore the importance of our observed positive correlation to a functional interaction between a nociceptive neurotransmitter, substance $\mathrm{P}$, and a pro-inflammatory cytokine, TNF $\alpha$, as one of the inflammatory processes perhaps underlying the development and/or presentation of CP.

From a knowledge translation perspective, the correlation difference between Preterm and Extremely Preterm Birth subgroups for TNF $\alpha$ and substance P may represent a potential interaction and intervention point between inflammation and nociceptive neurotransmission specifically in the CNS; however, demonstration of a spinal or trigeminal localization of this interaction would be required to underpin this interpretation. Such an understanding may provide an opportunity to improve outcomes through earlier intervention that includes targeting these specific pathways and their unique mechanisms. One weakness of the current analysis is that the group sizes are not robust enough to distinguish larger signature patterns/differences between groups that may be informational for identifying distinctions between different patient subgroup populations; ultimately, there may be different $\mathrm{CP}$ patient subgroups with specific, identifiable protein signatures. Larger samples with confirmatory analyses are needed. Additionally, further consideration regarding appropriate comparison groups or control samples is warranted. Healthy controls could be useful (see below regarding a point about establishing normative values) but their use could be limited in so much 


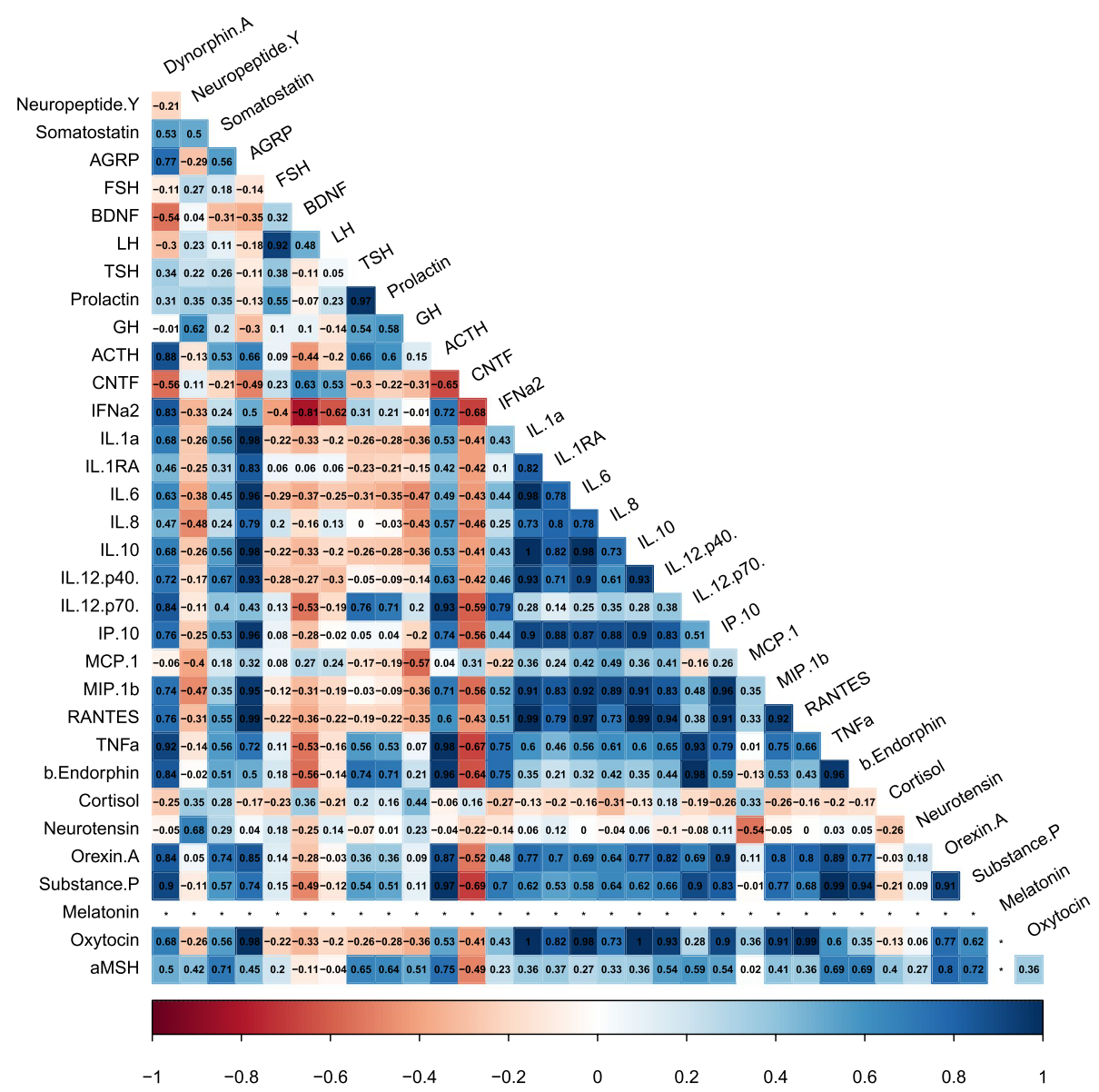

Fig. 4 Visual representation of the direction and strength of the Pearson's correlation coefficients between all 33 analytes assayed within the Extremely Preterm subgroup. Positive (blue), negative (red), strong (dark shading), and weak (light shading) correlations are depicted. *In instances where the same value was reported for each variable, no correlation was calculated

as it might be more important to consider inclusion of carefully defined samples from other neurodegenerative disorders in which more is known about the underlying immune pathology and disease progression (e.g., multiple sclerosis). Doing so would provide important points of comparison for similarities and differences in inflammatory analyte levels/profiles that would, in turn, increase our understanding of inflammatory mechanisms specific to the CP phenotype.

Our long-term goal is to establish clinical value in adopting a biomarker approach to understand clinical outcomes among patients with CP. A biological marker or biomarker is any characteristic that can be measured and evaluated as an indicator of normal biologic processes, pathologic processes, or pharmacologic responses to therapeutic intervention. Biomarkers hold the potential of a better understanding of the etiology and pathology of a given disorder, providing valuable insight into diagnosis, treatment, and prognosis for many debilitating disorders and diseases. Current diagnostic and therapeutic approaches to manage chronic disability among individuals with neurodevelopmental disorders including $\mathrm{CP}$ are limited by our narrow understanding of the biological mechanisms underlying developmental disorders of various etiologies and confounded further by phenotypic and etiologic heterogeneity (e.g., there is not one cause of CP; clinical presentation varies widely) and the lack of biomarkers predictive of therapeutic outcome.

Part of the hope is that molecular biomarkers may provide a useful 'work around' for closing the gap between clinical presentation and often unknown underlying pathophysiology in CP. To get there, however, there are enormous gaps in what is known from a normative perspective within the population of CP (e.g., there are few to no referent values for expected concentration values of the vast majority of inflammatory mediators). The difficulty with any clinical sample is whether the detectable analyte represents a biomarker for 'exposure' (to 
Table 4 Significant Pearson's correlations $(p \leq 0.001)$ between analyte pairs in the Extremely Preterm Birth subgroup. There were 24 unique positive analyte correlations (bold font) specific to the Extremely Preterm Birth subgroup that were not significant within the other gestational age subgroups

\begin{tabular}{|c|c|c|c|c|}
\hline Analyte 1 & Analyte 2 & Correlation & $95 \% \mathrm{Cl}$ & Adj. $p$ \\
\hline AGRP & IL-6 & 0.96 & {$[0.83,0.99]$} & 0.001 \\
\hline AGRP & $\mid \mathrm{P}-10$ & 0.96 & {$[0.81,0.99]$} & 0.001 \\
\hline AGRP & MIP-1 $\beta$ & 0.95 & {$[0.79,0.99]$} & 0.001 \\
\hline АСТН & $\beta$ endorphin & 0.96 & {$[0.81,0.99]$} & 0.001 \\
\hline IP-10 & MIP-1 $\beta$ & 0.96 & {$[0.80,0.99]$} & 0.001 \\
\hline TNFa & $\beta$ endorphin & 0.96 & {$[0.81,0.99]$} & 0.001 \\
\hline AGRP & IL-1a & 0.98 & {$[0.92,1.00]$} & $<.001$ \\
\hline AGRP & IL-10 & 0.98 & {$[0.92,1.00]$} & $<.001$ \\
\hline AGRP & RANTES & 0.99 & {$[0.95,1.00]$} & $<.001$ \\
\hline AGRP & Oxytocin & 0.98 & {$[0.92,1.00]$} & $<.001$ \\
\hline TSH & Prolactin & 0.97 & {$[0.86,0.99]$} & $<.001$ \\
\hline АСТН & TNFa & 0.98 & {$[0.92,1.00]$} & $<.001$ \\
\hline АCTH & substance $P$ & 0.97 & {$[0.88,0.99]$} & $<.001$ \\
\hline IL-1a & IL-6 & 0.98 & {$[0.92,1.00]$} & $<.001$ \\
\hline IL-1a & IL-10 & 1.00 & {$[1.00,1.00]$} & $<.001$ \\
\hline IL-1a & RANTES & 0.99 & {$[0.96,1.00]$} & $<.001$ \\
\hline IL-1a & Oxytocin & 1.00 & {$[1.00,1.00]$} & $<.001$ \\
\hline IL-6 & IL-10 & 0.98 & {$[0.92,1.00]$} & $<.001$ \\
\hline IL-6 & RANTES & 0.97 & {$[0.87,0.99]$} & $<.001$ \\
\hline IL-6 & Oxytocin & 0.98 & {$[0.92,1.00]$} & $<.001$ \\
\hline IL-10 & RANTES & 0.99 & {$[0.96,1.00]$} & $<.001$ \\
\hline IL-10 & Oxytocin & 1.00 & {$[1.00,1.00]$} & $<.001$ \\
\hline IL-12.p70 & $\beta$ endorphin & 0.98 & {$[0.92,1.00]$} & $<.001$ \\
\hline RANTES & Oxytocin & 0.99 & {$[0.96,1.00]$} & $<.001$ \\
\hline TNFa & substance $P$ & 0.99 & {$[0.97,1.00]$} & $<.001$ \\
\hline
\end{tabular}

an inflammatory process) or 'outcome' (of brain damage). There is no easy solution to this dilemma absent an experimental model. With that point acknowledged, we believe there is value in continuing to adopt and adapt a 'biomarker epidemiological' perspective as outlined by Dammann [5]. Doing so may help facilitate the development, testing, and application of immunomodulatory therapies for CP (see Fleiss and Gressens [8] for general considerations on this and related topics specific to tertiary management of brain damage as well as Lee et al. $2012[22])$.

In particular, it would be important to obtain comparison values from healthy and/or non-inflammatory based controls to establish the utility of neurochemical profile patterns as prognostic (natural history-like outcomes) or predictive tools (what profile would be most responsive to immune modulatory therapy trial). With that said, as noted above, careful selection of other well-defined diseases in which immune-mediated inflammation is a core feature would also be a valuable approach (e.g., multiple sclerosis - for which stem cell treatment trials are underway with a particular focus on patients with persistent inflammation [23]). A third approach - in a sense the strategy used for this preliminary work - is sampling within group (patient), but it would be strengthened considerably by larger samples and if/when ethically feasible, repeated measurement. Such a repeated measures approach was used by Koh et al. [24] in their investigation of cytokine changes in children with $\mathrm{CP}$ receiving intravenous granulocyte-colony stimulating factor followed by autologous mobilized peripheral blood mononuclear cells.

\section{Conclusion}

Given the preliminary data presented here: 1) that endocrine, neuropeptide, and inflammatory markers are detectable in CSF from pediatric patients with $\mathrm{CP}$ and 2) that significant correlations exist among markers for endocrine hormones, nociceptive neuropeptides and inflammatory mediators that are distinct in various subgroups of individuals with $\mathrm{CP}$, we think it is critical to continue this line of research to consider further the functional consequences of altered inflammatory processes and responses in children with CP and to consider the potential for mechanism-driven intervention based on protein/peptide/neurotransmitter signatures in $C P$ patients. Increasing our scientific understanding of the neurochemical milieu involved in distinct subgroups of $\mathrm{CP}$ may shed light on potential targets for earlier intervention to perhaps prevent transition to more severe presentation. Clarifying a mechanistic understanding of developmental differences may be what the analyte patterns are pointing to and offer a specific starting domain for further exploration. Our hope is that these preliminary findings lay the groundwork for additional studies to confirm and expand on the potential for protein signatures in $\mathrm{CP}$ to be valuable clinical tools in the future.

\footnotetext{
Abbreviations

CP: Cerebral palsy; CSF: Cerebral spinal fluid; WMD: White matter damage; TNFa: Tumor necrosis factor a; mRNA: Messenger ribonucleic acid; TLR4: Toll-like receptor 4; IRB: Institutional review board; ITB: Intrathecal baclofen; ELISA: Enzyme-linked immunosorbent assay; CRL: Cytokine reference laboratory; AgRP: Agouti-related peptide; FSH: Follicle-stimulating hormone; BDNF: Brain-derived neurotrophic factor; LH: Luteinizing hormone; TSH: Thyroid stimulating hormone; GH: Growth hormone; ACTH: Adrenocorticotropic hormone; PRL: Prolactin; CNTF: Human ciliary neurotropic factor; IFNa2: Interferon alpha 2; IL-1 a: Interleukin 1 alpha; IL-1 ra: Interleukin 1 receptor antagonist; IL-6: Interleukin 6; IL-8: Interleukin 8; IL-10: Interleukin 10; IL-12p40: Interleukin 12 subunit 40; IL-12p70: Interleukin 12 subunit 70; IP-10: Interferon gamma-induced protein 10; MCP-1: Monocyte chemotactic protein 1; MIP1 $\beta$ : Macrophage inflammatory protein 1 beta; RANTES: Regulated on activation normal T expressed and secreted; TNFa: Tumor necrosis factor alpha; a-MSH: Melanocyte-stimulating hormone; DynA: Dynorphin A; PE: Phycoerythrin; Corr:
} 
Correlation; Cl: Confidence Interval; Adj P: Adjusted P; M: Mean; SD: Standard Deviation; rpm: Revolutions per minute; $\mu$ L: Microliters; GM-CSF: Granulocytemacrophage colony-stimulating factor; $\mathrm{PGE}_{2}$ : Prostaglandin E2; FDA: Food and Drug Administration; IL1R1: IL-1 receptor 1; OX1R: Orexin 1 receptor; OX2R: Orexin 2 receptor; NK1R: Neurokinin 1 receptor; Gq: Gq protein-coupled alpha subunit receptor; ${ }^{\circ} \mathrm{C}$ : Degrees Celsius.

\section{Supplementary Information}

The online version contains supplementary material available at https://doi. org/10.1186/s12883-021-02333-2.

Additional file 1: Table 1. A Pearson's correlation test against the null hypothesis of 0 was conducted for all possible pairs of analytes assayed, controlling for familywise Type I errors using the Benjamini \& Hochberg (1995) false discovery rate correction. As there were 528 correlation tests, only those retaining a significant correlation $(p \leq 0.05)$ after the false discovery rate correction are presented. Table 2. Pearson's correlation coefficients between pairs of analytes within the Term Birth gestational age subgroup. Only significant correlations $(p \leq 0.05)$ after controlling for false discovery rate are presented. There were no unique analyte correlations specific to those with Term Birth that were not also significant within the other gestational age subgroups. Table 3. Pearson's correlation coefficients between pairs of anlytes within the Preterm Birth gestational age subgroup. Only significant correlations $(p \leq 0.05)$ after controlling for false discovery rate are presented. Table 4. Pearson's correlation coefficients between pairs of analytes within the Extremely Preterm Birth gestational age subgroup. Only significant correlations $(p \leq 0.05)$ after controlling for false discovery rate are presented. Figure 1. Visual representation of the direction and strength of the Pearson's correlation coefficients between analytes assayed within the subgroup with spastic CP. Positive (blue), negative (red), strong (dark shading), and weak (light shading) correlations are depicted. Table 5A. Pearson's correlation coefficients between pairs of analytes within the subgroup with spastic CP. Complete dataset of significant correlations $(p \leq 0.05)$ after controlling for false discovery rate are presented. Table 5B. Significant Pearson's correlations ( $p \leq 0.001)$ between analyte pairs in the subgroup with spastic CP. There were 8 unique positive analyte correlations (bold font) specific to spastic CP that were not significant within the subgroup with mixed tone CP. Figure 2 . Visual representation of the direction and strength of the Pearson's correlation coefficients between analytes assayed within the subgroup with mixed tone CP. Positive (blue), negative (red), strong (dark shading), and weak (light shading) correlations are depicted. Table 6A. Pearson's correlation coefficients between pairs of analytes within the subgroup with mixed tone CP. Complete dataset of significant correlations $(p \leq 0.05)$ after controlling for false discovery rate are presented. Table 6B. Significant Pearson's correlations $(p \leq 0.001)$ between analyte pairs in the subgroup with mixed tone CP. There were 7 unique positive analyte correlations (bold font) specific to mixed tone CP that were not significant within the subgroup with spastic CP. Figure 3. Visual representation of the direction and strength of the Pearson's correlation coefficients between analytes assayed within the subgroup with seizures present. Positive (blue), negative (red), strong (dark shading), and weak (light shading) correlations are depicted. Table 7A. Pearson's correlation coefficients between pairs of analytes within the subgroup with seizures present. Complete dataset of significant correlations $(p \leq 0.05)$ after controlling for false discovery rate are presented. Table 7B. Significant Pearson's correlations $(p \leq 0.001)$ between analyte pairs in the subgroup with seizures. There were no unique analyte correlations specific to those with seizures that were not also significant within the subgroup without seizures. Figure 4. Visual representation of the direction and strength of the Pearson's correlation coefficients between analytes assayed within the subgroup without seizures. Positive (blue), negative (red), strong (dark shading), and weak (light shading) correlations are depicted. Table $\mathbf{8 A}$. Pearson's correlation coefficients between pairs of analytes within the subgroup without seizures. Complete dataset of significant correlations $(p \leq 0.05)$ after controlling for false discovery rate are presented. Table $\mathbf{8 B}$. Significant Pearson's correlations $(p<0.001)$ between analyte pairs in the subgroup without seizures. There were 30 unique positive analyte correlations (bold font) specific to those without seizures that were not significant within the subgroup with seizures. Figure 5. Visual representation of the direction and strength of the Pearson's correlation coefficients between analytes assayed within the subgroup with quadriplegia. Positive (blue), negative (red), strong (dark shading), and weak (light shading) correlations are depicted. Table 9A. Pearson's correlation coefficients between pairs of analytes within the subgroup with quadriplegia. Complete dataset of significant correlations $(p \leq 0.05)$ after controlling for false discovery rate are presented. Table 9B. Significant Pearson's correlations $(p \leq 0.001)$ between analyte pairs in the subgroup with quadriplegia. There were 16 unique positive analyte correlations (bold font) specific to those with quadriplegia that were not significant within the subgroup without quadriplegia. Figure 6. Visual representation of the direction and strength of the Pearson's correlation coefficients between analytes assayed within the subgroup without quadriplegia. Positive (blue), negative (red), strong (dark shading), and weak (light shading) correlations are depicted. Table 10A. Pearson's correlation coefficients between pairs of analytes within the subgroup without quadriplegia. Complete dataset of significant correlations $(p \leq 0.05)$ after controlling for false discovery rate are presented. Table 10B. Significant Pearson's correlations $(p \leq 0.001)$ between analyte pairs in the subgroup without quadriplegia. There were 2 unique positive analyte correlations (bold font) specific to those without quadriplegia that were not significant within the subgroup with quadriplegia.

\section{Acknowledgements}

Not applicable.

\section{Authors' contributions}

CGP: statistical design, data analysis, manuscript writing. CB: study design, implementation, manuscript writing. APM: study design, analyses, manuscript review. ME: specimen processing, specimen analyses, manuscript writing. GW: manuscript writing, manuscript review. PG: study implementation, recruitment/specimen procurement oversight, manuscript review. MP: study conception, recruitment/specimen procurement oversight, manuscript review. FS: study conception, design, manuscript writing. The author(s) read and approved the final manuscript.

\section{Funding}

This study was funded, in part, by Eunice Kennedy Shriver NICHD Grant No. 73126.

\section{Availability of data and materials}

All data generated or analyzed during this study are included in this published article [and its Supplementary information file]. The datasets analyzed are available from the corresponding author on reasonable request.

\section{Declarations}

Ethics approval and consent to participate

All work described was approved by the Institutional Review Board (IRB) of the University of Minnesota. All participants provided written consent in accordance with IRB consent form review and approval. Committee reference number 0809M46301.

\section{Consent for publication}

Not applicable. All data is aggregate.

\section{Competing interests}

The authors declare that they have no competing interests.

\section{Author details}

${ }^{1}$ College of Pharmacy, University of Minnesota, Minneapolis, MN 55455, USA ${ }^{2}$ Gillette Children's Specialty Healthcare, Saint Paul, MN 55101, USA. ${ }^{3}$ Department of Pediatrics, University of Minnesota Medical School, Minneapolis, MN 55455, USA. ${ }^{4}$ Departments of Neuroscience, Pharmacology, Dermatology, University of Minnesota Medical School, Minneapolis, MN 55455, USA. ${ }^{5}$ Children's Mercy, Kansas City, MO 64108, USA. ${ }^{6}$ Department of Educational Psychology, College of Education and Human Development, Minneapolis, MN 55455, USA. 
Received: 29 May 2020 Accepted: 23 July 2021

Published online: 04 October 2021

\section{References}

1. Kaukola T, et al. Cerebral palsy is characterized by protein mediators in cord serum. Ann Neurol. 2004;55(2):186-94.

2. Hagberg H, Gressens P, Mallard C. Inflammation during fetal and neonatal life: implications for neurologic and neuropsychiatric disease in children and adults. Ann Neurol. 2012;71(4):444-57.

3. Volpe JJ. Brain injury in premature infants: a complex amalgam of destructive and developmental disturbances. Lancet Neurol. 2009;8(1):110-24.

4. Douglas-Escobar M, Weiss MD. Biomarkers of brain injury in the premature infant. Front Neurol. 2012;3:185.

5. Dammann O, Leviton A. Inflammatory brain damage in preterm newborns-dry numbers, wet lab, and causal inferences. Early Hum Dev. 2004;79(1):1-15.

6. Neufeld MD, et al. Maternal infection and risk of cerebral palsy in term and preterm infants. J Perinatol. 2005;25(2):108-13.

7. Dammann O. Persistent neuro-inflammation in cerebral palsy: a therapeutic window of opportunity? Acta Paediatr. 2007;96(1):6-7.

8. Fleiss B, Gressens P. Tertiary mechanisms of brain damage: a new hope for treatment of cerebral palsy? Lancet Neurol. 2012;11(6):556-66.

9. Lin CY, et al. Altered inflammatory responses in preterm children with cerebral palsy. Ann Neurol. 2010;68(2):204-12.

10 Leviton A, et al. The relationship between early concentrations of 25 blood proteins and cerebral white matter injury in preterm newborns: the ELGAN study. J Pediatr. 2011;158(6):897-903.e1-5.

11. Zareen Z, et al. Cytokine dysregulation in children with cerebral palsy. Dev Med Child Neurol. 2021;63(4):407-12.

12. Roberson $R$, et al. Postnatal inflammatory rat model for cerebral palsy: too different from humans. Am J Obstet Gynecol. 2006;195(4):1038-44.

13. Palisano $R$, et al. Development and reliability of a system to classify gross motor function in children with cerebral palsy. Dev Med Child Neurol. 1997;39(4):214-23.

14. de Lecea L. Cortistatin: a natural somatostatin analog. J Endocrinol Invest. 2005;28(11 Suppl International):10-4.
15. De Koninck Y, Henry JL. Substance P-mediated slow excitatory postsynaptic potential elicited in dorsal horn neurons in vivo by noxious stimulation. Proc Natl Acad Sci U S A. 1991;88(24):11344-8.

16. Todd AJ. Anatomy of primary afferents and projection neurones in the rat spinal dorsal horn with particular emphasis on substance $P$ and the neurokinin 1 receptor. Exp Physiol. 2002;87(2):245-9.

17. Thome JG, et al. Contributions of interleukin-1 receptor signaling in traumatic brain injury. Front Behav Neurosci. 2019;13:287.

18. Bårdsen K, et al. Interleukin-1-related activity and hypocretin-1 in cerebrospinal fluid contribute to fatigue in primary Sjögren's syndrome. J Neuroinflammation. 2019;16(1):102.

19. Strittmatter $M$, et al. CSF substance $P$ somatostatin and monoaminergic transmitter metabolites in patients with narcolepsy. Neurosci Lett. 1996;218(2):99-102.

20. American Academy of Sleep Medicine. The international classification of sleep disorders : diagnostic and coding manual. 2nd ed. 2005, Westchester: American Academy of Sleep Medicine. xviii, p. 297.

21. Palma $C$, et al. Functional characterization of substance $P$ receptors on cultured human spinal cord astrocytes: synergism of substance $P$ with cytokines in inducing interleukin-6 and prostaglandin E2 production. Glia. 1997;21(2):183-93.

22. Lee $\mathrm{YH}$, et al. Safety and feasibility of countering neurological impairment by intravenous administration of autologous cord blood in cerebral palsy. J Transl Med. 2012;10:58.

23. Uccelli A, et al. MEsenchymal StEm cells for Multiple Sclerosis (MESEMS): a randomized, double blind, cross-over phase I/II clinical trial with autologous mesenchymal stem cells for the therapy of multiple sclerosis. Trials. 2019;20(1):263.

24. Koh $\mathrm{H}$, et al. Serial changes of cytokines in children with cerebral palsy who received intravenous granulocyte-colony stimulating factor followed by autologous mobilized peripheral blood mononuclear cells. J Korean Med Sci. 2018;33(21):e102

\section{Publisher's Note}

Springer Nature remains neutral with regard to jurisdictional claims in published maps and institutional affiliations.
Ready to submit your research? Choose BMC and benefit from:

- fast, convenient online submission

- thorough peer review by experienced researchers in your field

- rapid publication on acceptance

- support for research data, including large and complex data types

- gold Open Access which fosters wider collaboration and increased citations

- maximum visibility for your research: over $100 \mathrm{M}$ website views per year

At BMC, research is always in progress.

Learn more biomedcentral.com/submissions 